\title{
Drone-Based Daylight Electroluminescence Imaging of PV Modules
}

Benatto, Gisele Alves dos Reis; Mantel, Claire; Spataru, Sergiu Viorel; Santamaria Lancia, Adrian Alejo; Riedel, Nicholas; Thorsteinsson, Sune; Poulsen, Peter; Parikh, Harsh Rajesh; Forchhammer, Søren; Séra, Dezso

\section{Published in:}

IEEE Journal of Photovoltaics

Link to article, DOI:

10.1109/JPHOTOV.2020.2978068

Publication date:

2020

Document Version

Peer reviewed version

Link back to DTU Orbit

Citation (APA):

Benatto, G. A. D. R., Mantel, C., Spataru, S. V., Santamaria Lancia, A. A., Riedel, N., Thorsteinsson, S. Poulsen, P., Parikh, H. R., Forchhammer, S., \& Séra, D. (2020). Drone-Based Daylight Electroluminescence Imaging of PV Modules. IEEE Journal of Photovoltaics, 10(3), 872 - 877.

https://doi.org/10.1109/JPHOTOV.2020.2978068

\section{General rights}

Copyright and moral rights for the publications made accessible in the public portal are retained by the authors and/or other copyright owners and it is a condition of accessing publications that users recognise and abide by the legal requirements associated with these rights.

- Users may download and print one copy of any publication from the public portal for the purpose of private study or research.

- You may not further distribute the material or use it for any profit-making activity or commercial gain

- You may freely distribute the URL identifying the publication in the public portal 


\title{
Drone-Based Daylight Electroluminescence Imaging of PV Modules
}

\author{
Gisele A. dos Reis Benatto ${ }^{1}$, Claire Mantel $^{1}$, Sergiu Spataru ${ }^{1}$, Adrian A. Santamaria Lancia ${ }^{1}$, Nicholas \\ Riedel $^{1}$, Sune Thorsteinsson ${ }^{1}$, Peter B. Poulsen ${ }^{1}$, Harsh Parikh ${ }^{2}$, Søren Forchhammer ${ }^{1}$, Dezso Sera ${ }^{3}$ \\ ${ }^{1}$ Department of Photonics Engineering, Technical University of Denmark, Frederiksborgvej 399, 4000, \\ Roskilde, Denmark.
}
${ }^{2}$ Department of Energy Technology, Aalborg University, Pontoppidanstraede 101, 9220, Aalborg, Denmark. ${ }^{3}$ Science and Engineering Faculty, Queensland University of Technology, Gardens Point Campus, 2 George Street, QLD 4000, Brisbane, Australia.

\begin{abstract}
Electroluminescence (EL) imaging is a PV module characterization technique, which provides high accuracy in detecting defects and faults such as cracks, broken cells interconnections, shunts, among many others; furthermore, the EL technique is used extensively due to a high level of detail and direct relationship to injected carrier density. However, this technique is commonly practiced only indoors - or outdoors from dusk to dawn - because the crystalline silicon luminescence signal is several orders of magnitude lower than sunlight. This limits the potential of such a powerful technique to be used in utility scale inspections, and therefore the interest in the development of electrical biasing tools to make outdoor EL imaging truly fast and efficient. With the focus of quickly acquiring EL images in daylight, we present in this article a drone-based system capable of acquiring EL images at a framerate of $\mathbf{1 2 0}$ frames per second. In a single second during high irradiance conditions, this system can capture enough EL and background image pairs to create an EL PV module image that has sufficient diagnostic information to identify faults associated with power loss. The final EL images shown in this

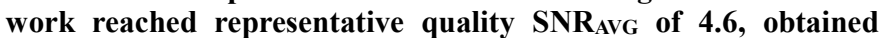
with algorithms developed in previous works. These drone-based EL images were acquired with global horizontal solar irradiance close to one sun in the plane of the array.
\end{abstract}

Index Terms-Electroluminescence, Imaging, Photovoltaic cells, Crystalline Silicon PV, Characterization of Defects in PV.

\section{INTRODUCTION}

In 2017, the worldwide photovoltaic electricity production was of $460 \mathrm{TWh}$, with a cumulative capacity of almost 400 GWp [1]. This growth represents a significant investment in the energy sector, in the order of a hundred billion dollars or euros. Approximately $60 \%$ of the world's PV capacity corresponds to utility-scale projects and this share has only increased in the last years. This trend points out the need for fast and effective PV inspections to assure the expected solar energy harvest.

The accuracy in identifying module faults in a PV plant is usually proportional to the time dedicated to the inspection. Fast inspections can be performed with Unmanned Aerial Vehicles (UAV, or drones) using visual and thermographic cameras, which provide information on glass breakage, soiling, bird dropping, disconnected strings, hotspots, among other major defects. However, disconnected PV modules or strings, PID shunted cells, and faulty bypass diodes are all failures that are not detectable by visual inspection and cause only a slight temperature increase between active and inactive module parts, making them more difficult or even impossible to detect under low irradiance conditions [2]. Electroluminescence (EL) is inherently more accurate than infrared thermography in identification of faults because EL images offer resolution in the semiconductor material level. Furthermore, the availability of EL is significantly higher than infrared, considering weather conditions vary greatly with location and latitude [3]. The limitations of EL imaging mainly occur due to the sensitivity of the camera detector in the wavelength of interest and the presence of intense stray light, essentially sunlight.

The recently published IEC technical specification for EL and field experience require EL measurements to be acquired under $<100 \mathrm{~W} \mathrm{~m}^{-2}$ outdoor light intensity [2], [4]. Such a constraint not only limits inspection time, but also can create additional drawbacks, for instance restrictions to site access, UAV flight time restrictions, and safety concerns when making the electrical connections in a dark and sometimes humid environment, among others.

It is of high importance to develop a system that can perform daylight EL with UAV image acquisition. Under bright sunlight (i.e. global horizontal irradiance - GHI $>100$

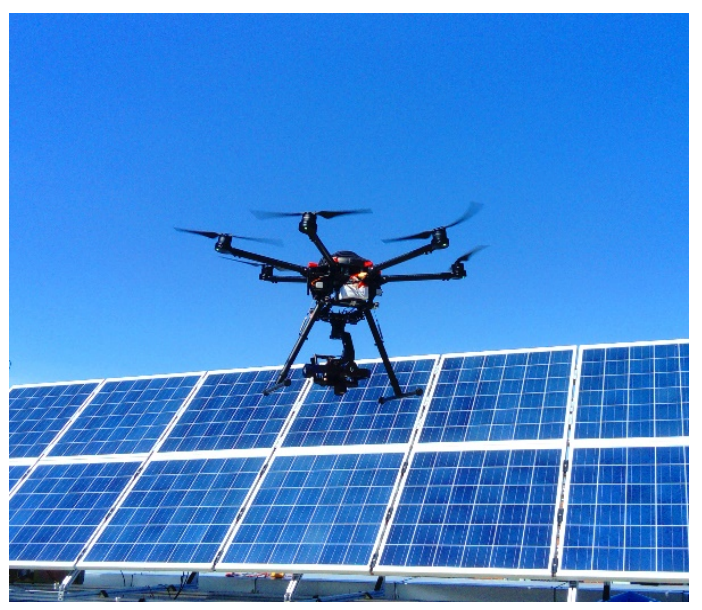

Fig. 1. Drone-based daylight EL acquisition system. 
$\mathrm{W} \mathrm{m}^{-2}$ ) it is usually not sufficient to use daylight filters and subtract (calculate the difference of each pixel) a single background (BG) image to obtain a clear EL image. The intensity of the EL signal is several orders of magnitude lower than sunlight, which lead to a very low signal-to-noise ratio (SNR) [5], [6]. However, by acquiring many EL/BG image pairs taken at the same fixed position and adding post processing steps - which consist on the average and subtraction of the BG for each pair - the SNR can be increased to an acceptable level. For outdoor EL, the accepted $\mathrm{SNR}_{50}$ (the simplified quantification of image quality) should be higher than 5 [4]. In addition, an extension of the $\mathrm{SNR}_{50}$ equation was proposed for the specific case when averaging is required [7], where $\mathrm{SNR}_{\mathrm{AVG}}$ should also be higher than 5 to obtain an outdoor EL image with satisfactory quality.

The primary requirement for daylight EL imaging is that images have to be acquired fast and with short exposure times to avoid motion blur and saturation due to sunlight; this requirement can be met with the use of cameras with InGaAs based detectors [8]. Even with the appropriate hardware, there are several further challenges to enhance the SNR of images taken from a UAV system: i) each PV panel has to be segmented with high accuracy; ii) the motion of the UAV must be compensated for the images to be averaged without loss in quality; iii) the true EL/BG difference - that can be translated in a delta of the mean pixel values - must remain stable and substantial in magnitude.

Here we present an acquisition system installed in a drone (Fig. 1) able to acquire $120 \mathrm{EL} / \mathrm{BG}$ modulated images per second (i.e. 60 image pairs), allowing bright daylight EL imaging of PV modules. The modulation approach (sequential acquisition of EL/BG image pairs) was adopted instead of a batch approach (sequential acquisition of EL images and then sequential acquisition of BG images), as modulation was a more robust approach during high solar irradiance variability, which is often the case in Denmark where the test flights were performed. The modulated electrical waveform was applied using two separate approaches: DC square waveform and $\mathrm{AC}+\mathrm{DC}$ sinusoidal waveform. The final drone-based EL images shown in this work presented lower quality compared to those obtained indoors and stationary in daylight, but still having sufficient quality to identify the main features related to the module power loss.

\section{METHODOLOGY}

In the following subsections, the details of the image acquisition system, device under test (DUT), electrical modulation and image post processing are presented. These steps were employed for the obtainment of the final EL images from a drone.

\section{A. Image acquisition}

The EL/BG image pair acquisition apparatus is comprised of a hexacopter drone equipped with an InGaAs camera (640 x 512 pixels sensor) and an embedded PC for camera control, live image feedback and image storage. Fig. 2 shows an overlay of the solar spectrum, filter transmission and sensor sensitivity, which are the elements present in a typical daylight EL acquisition. The EL signal corresponds to the crystalline silicon (c-Si) luminescence spectrum. The camera, lens and filter details have been presented previously [9]. The acquired image consisted in the luminescence spectra from c-Si (red line) and sunlight (here the AM 1.5 spectrum - black line) which for this case is considered as noise. The spatial EL acquisition was made by an InGaAs sensor with quantum efficiency (QE) at around $80 \%$ in the region of the c-Si peak luminescence. To avoid the acquisition of the sun irradiance in most of the sensor $\mathrm{QE}$ region and favor the c-Si acquisition, a bandpass (BP) filter was used and its transmittance is also represented in Fig. 2. During the sequence acquisition, the exposure time must be kept fixed and chosen at the measurement to avoid saturation due to the sunlight.

Fig. 3 shows the main controls and hardware involved in the flight and image acquisition, as well as the post processing steps performed after flight. The drone and camera control were connected to a computer via two different Wi-Fi channels for better signal stability. The drone controller software allowed semi-autonomous flights, according to regulations on safety. The images are all stored locally in the

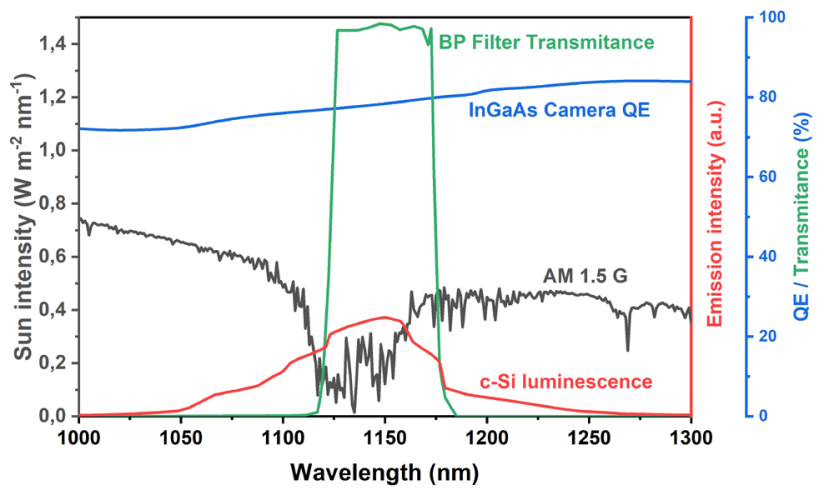

Fig. 2. Signal and sensor involved in daylight EL imaging. See text for further details.

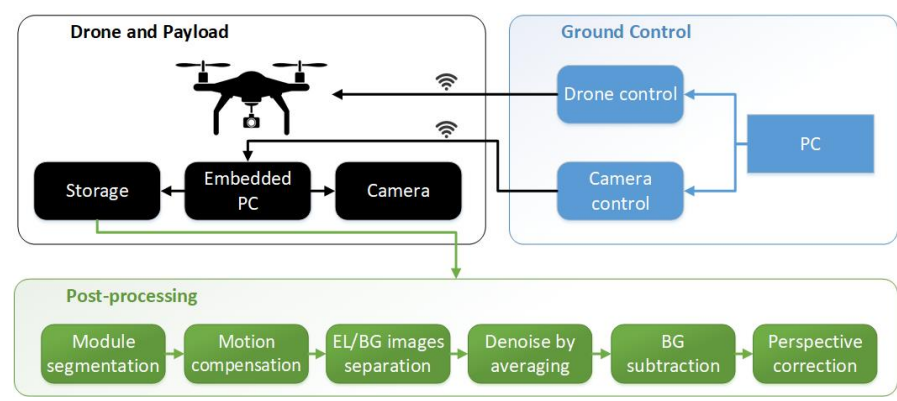

Fig. 3. Components of the drone-based EL/BG image acquisition system and post processing steps. 
embedded PC (Apalis TK1 from Toradex) [10] and used for post image processing afterwards.

\section{B. DUT and electrical modulation}

A mechanically stressed PV module with 36 multicrystalline $15.6 \times 15.6 \mathrm{~cm}$ cells arranged in a $6 \times 6$ cells matrix is used as the DUT in this work. Every two columns of the DUT were connect to a bypass diode, therefore the device had 3 bypass diodes in total. As informed by the manufacturer, $\mathrm{V}_{\mathrm{OC}}$ and $\mathrm{I}_{\mathrm{SC}}$ under STC conditions of this particular module was $22.6 \mathrm{~V}$ and $8.4 \mathrm{~A}$ respectively. The stressed module contained cells with several electrically connected cracks (A cracks), partially (B cracks) and completely isolated regions (C cracks) [11].

In order to witness the waveform shape and frequency flowing through the DUT during the EL image sequence acquisition, the measurement circuit illustrated in Fig. 4 was arranged to monitor the electrical signal in the PV module. The measurement setup consisted of a power supply unit (PSU) and a Keysight DSO-X2004A, 4-channel oscilloscope. The power supply was connected directly to the terminals of the DUT, and a $10 \mathrm{~m} \Omega$ shunt resistor was placed at the negative terminal. The DUT voltage $\mathrm{V}_{\mathrm{PV}}$ was measured at the terminals of the DUT and the current was measured as the voltage drop $V_{\text {Rsh }}$ across the shunt resistor. Both voltages were measured in differential mode and the absolute value was calculated out of the subtraction of the positive and negative values for each $V_{\text {Rsh }}$ and $V_{P V}$.

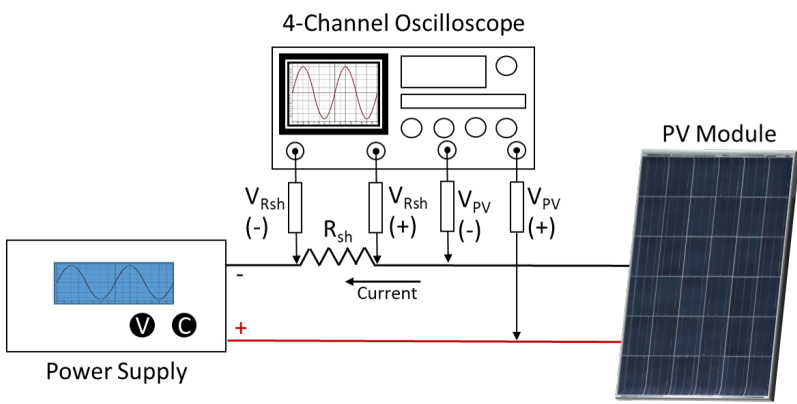

Fig. 4. Measurement circuit for the PSU waveforms running in the PV module.

\section{a. DC electrical modulation}

A bidirectional DC power supply unit (PSU) SM 1500-CP30 from Delta Elektronika [12] was used to generate a modulated voltage bias for the PV panels, with a period chosen as a multiple of the camera acquisition period. Fig. 5 shows the measured voltage and current waveform programmed at $60 \mathrm{~Hz}$ when the connected PV panel was indoors at low light conditions and outdoors under $970 \mathrm{~W} \mathrm{~m}^{-2}$ global irradiance measured in the plane of the array $\left(\mathrm{G}_{\mathrm{POA}}\right)$; the secondary $\mathrm{y}$-axis shows the mean pixel values of the PV

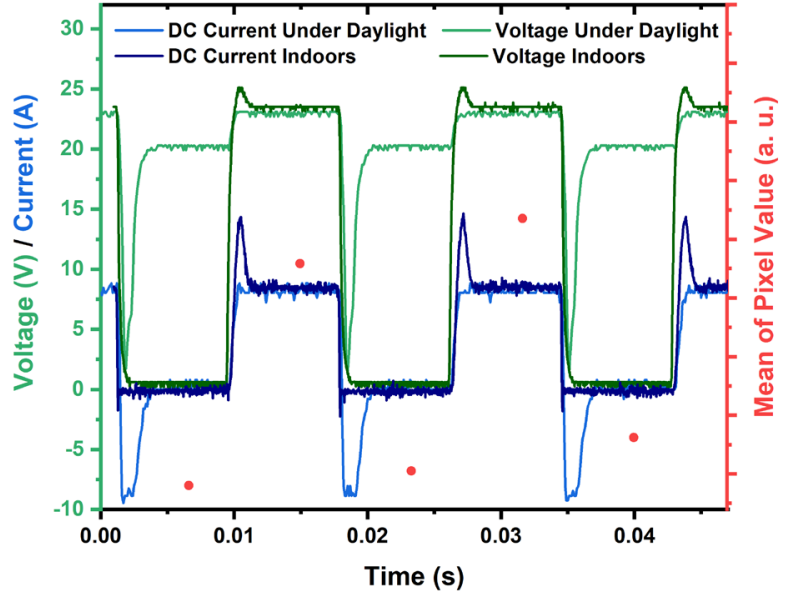

Fig. 5. DC current waveform at $60 \mathrm{~Hz}$ flowing through a PV panel indoors under low light and outdoors under $970 \mathrm{~W} \mathrm{~m}^{-2}$ in the plane of the array.

panel EL/BG image pairs acquired at $120 \mathrm{fps}$ outdoors at the same time.

The PSU was programmed to be controlled by voltage, corresponding current limits were set to $0 \mathrm{~A}$ and STC ISC. The DC square wave signal was programmed with a period of 16.67 milliseconds, i.e. frequency of $60 \mathrm{~Hz}$. The forward bias applied to the PV consisted of a programmed square wave DC voltage signal, pulsing between $0 \mathrm{~V}$ and $25 \mathrm{~V}$. During the current and voltage modulation, it can be seen that indoors (no sun irradiance), the voltage goes to zero and tries to go the 25 $\mathrm{V}$, but switches automatically to current control (CC) mode in $\sim \mathrm{I}_{\mathrm{SC}}$ and correspondent voltage (slightly higher then $\mathrm{V}_{\mathrm{OC}}$ ). This can be seen as a brief (app. $1 \mathrm{~ms}$ ) transient overshoot in the current square wave measured indoor. Note that the panel voltage at the same injected current is slight lower than in the indoors case as the solar panel temperature was few degrees higher during the daylight. When the bias is performed outdoors, the programmed period for the voltage goes to $0 \mathrm{~V}$ results in a brief overshoot to $-I_{\mathrm{SC}}$, as expected due to the sun irradiation on the device. Shortly after, the PSU switches automatically to $\mathrm{CC}$ mode, keeps the voltage at $\mathrm{V}_{\mathrm{OC}}$ and the current closely to $0 \mathrm{~A}$ following the programmed waveform. When the voltage goes to $25 \mathrm{~V}$, the PSU holds it in CC mode, avoiding the voltage provoked overshoot and maintaining the current at forward bias ISC and correspondent voltage close to $V_{\text {OC. }}$ Such effects can be explained by the PSU programming be performed based on voltage control, current limits driven by the PV module IV characteristics and capacitive effects of the cells in the module.

From the luminescence signal point of view and in general terms in module level in the purpose of this work, the luminescence intensity is directly proportional to the (internally and externally) injected carrier density in the PV device [13], [14]. Considering this, the mean pixel value of the $\mathrm{PV}$ panel images is higher when the injected current is higher, and lower when current is zero. However, the luminescence 
signal is not zero when the injected current is zero under daylight conditions, as photoluminescence (PL) occurs between $\mathrm{V}_{\mathrm{OC}}$ and $\mathrm{V}_{\mathrm{MPP}}$, with virtually zero PL signal at - $\mathrm{I}_{\mathrm{SC}}$ [13], [15], [16]. Here we consider that "PL only" image acquisition would occur if images taken at $\mathrm{V}_{\mathrm{OC}}$ were subtracted by images obtained at the -ISC current overshoot, but the probability of the images to be acquired under the former condition was low and not observed during our tests in the form of abnormally lower mean pixel values. The collection of EL/BG images performed in the DC modulation condition was correspondent of the electrical signal in the DUT being $\mathrm{I}_{\mathrm{SC}}$ and zero current respectively. Therefore, PL signal is taken out by the subtraction, as the "low pixel value" images - considered here BG images - contain most of the PL signal that is not accounted for in the final version of the image. Thus, the BG images here are not as typically used for EL imaging background images during nighttime or indoors (no luminescence signal), as for daylight conditions the BG images do contain PL information. In the future, the consideration of the valuable characterization information contained in PL images and its potential to increase the overall luminescence signal should be considered for the DC modulation scenario. Here, only the EL image type of information was chosen to be considered, especially for the final image qualitative interpretation.

The frequencies of the electrical bias and framerate result in collecting an EL/BG image pair one after the other. This approach minimizes the interference of rapid fluctuations in solar irradiance during the measurements due to different levels of cloud cover. The apparent rise of the pixel values over time in Fig. 5 is an example of the effect of such interference, already noticeable in such short time period. The fast framerate allows acquiring many images of the same PV panel with the drone in movement, thus permitting the acquisition of several sequences of images (or video with several scenes) without the need for brief stops in front of each module. As the camera was not actively synchronized with the bias signal, an additional step separating EL from BG images by post-processing was necessary. This postprocessing uses the average pixel value within the panel (higher for EL than BG images). The absence of synchronization between the camera and PSU implies that the phase between image acquisition and bias signal is not guaranteed and the post-processing also had to verify that the average pixel value between EL and BG was large enough to create a useful difference image. EL image repeatability without synchronization with the modulation signal can be achieved by EL image oversampling, analysis of the high and low states, and discarding the transient states. For the DC electrical signal, the measured frequency showed to be lower than expected compared to the programmed modulation period $(\sim 58 \mathrm{~Hz})$ due to limitations of the DC power supply control. This lower frequency leads to a mismatch with the camera acquisition frequency. Consequently, a significant amount of images acquired in the sequence are discarded as transient states by the EL/BG separation algorithm, leaving less images available for averaging and quality enhancement.

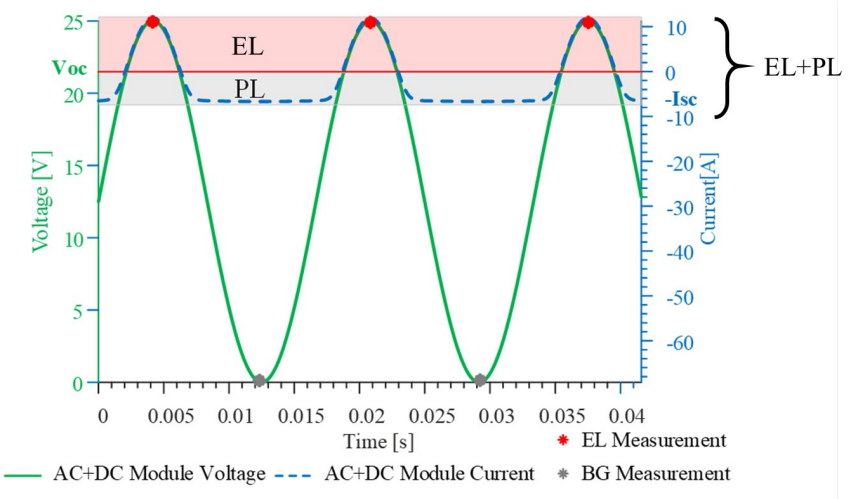

Fig. 6. Simulated $\mathrm{AC}+\mathrm{DC}$ current and voltage waveform at $60 \mathrm{~Hz}$ under $850 \mathrm{~W} \mathrm{~m}^{-2}$ and $48{ }^{\circ} \mathrm{C}$ cell temperature, depicting the $\mathrm{PL}$ (gray), EL (red) operation regions, and time instances when EL signal and $\mathrm{BG}$ images are acquired.

\section{b. AC+DC electrical modulation}

An AC PSU model AC6804A from Keysight was also used for modulated bias to avoid strong sun irradiance interferences in the bias signal. The forward bias applied to the PV consisted of an $\mathrm{AC}+\mathrm{DC}$ coupling signal (sinusoidal AC signal with a DC offset). As in the DC case, the $\mathrm{AC}+\mathrm{DC}$ sinusoidal signal frequency was $60 \mathrm{~Hz}$, while image acquisition was performed with a framerate of $120 \mathrm{~Hz}$. A simulation of the voltage and current waveform under sunlight $\left(850 \mathrm{~W} \mathrm{~m}^{-2}\right.$ and $48{ }^{\circ} \mathrm{C}$ cell temperature, is shown in Fig. 6. Here we can observe in gray the $\mathrm{I}_{\mathrm{SC}}$ to $\mathrm{V}_{\mathrm{OC}}$ operation region of the module, where the solar cell emit PL due to sunlight excitation only. The PL intensity is minimal at the $\left(0 \mathrm{~V},-\mathrm{I}_{\mathrm{SC}}\right)$ operation point, since most of the excess carriers are extracted [13], [14].

As in the previous case described in section B.a, the absence of synchronization between the camera and PSU implies that the phase between image acquisition and the waveform shown in Fig. 6 is arbitrary and verified by image post-processing. For this waveform, the delta can vary between acquisition sequences and is not necessarily dependent of the sun irradiation. A delta indicated by post-processing assures that the EL/BG difference is enough to acquire EL images correspondent to high (EL) and no/low bias (BG) states. The $\mathrm{AC}+\mathrm{DC}$ modulation signal is smoother and frequency stable compared to the DC modulation, so as soon as the image acquisition starts close to a maximum or minimum bias, all the images in the sequence are available for image enhancement. More image sequences might be required to reach repeatability of EL imaging using $\mathrm{AC}+\mathrm{DC}$ modulation, as the start point of the acquisition is arbitrary. 
Between $\mathrm{V}_{\mathrm{OC}}(21.5 \mathrm{~V})$ and $\mathrm{V}_{\mathrm{MAX}}(25 \mathrm{~V})$, depicted in red in Fig. 6, the module is forward biased, and current is injected in the solar cells, causing EL radiation in addition to PL, as in this case the BG image does contain PL signal. The maximum EL radiation is reached at the maximum injected current (shown in the example of Fig. 6). As the acquisition for each operation point is arbitrary, the luminescence signal captured is EL+PL or can even be PL only, but for the sake of the comparison in this paper, we will call it "EL image".

\section{Post processing}

After drone flights, the images are transferred from the embedded PC internal storage and post processed using algorithms developed in MATLAB. The image processing steps for stationary daylight EL include EL/BG image separation, averaging, BG subtraction, perspective correction of the obtained image following the procedure detailed in [17], and quality control, i.e. $\mathrm{SNR}_{\mathrm{AVG}}$ calculation following the formula detailed in [7]. For EL/BG images acquired in movement, as it is the case with drone acquisition, the following additional steps must be performed: i) PV panel edge detection and segmentation in each image of the sequence; ii) motion compensation of the whole image sequence. These two steps are performed before the EL/BG image separation, which is the first step for stationary daylight EL.

These additional steps require a very high degree of accuracy and robustness of the image processing algorithms, adding a much higher level of complexity. Moreover, EL/BG separation needs also to be improved to overcome variations not only from occasional cloud cover, but also from changes of angle of acquisition.

In each frame, the panel is firstly detected and segmented from the rest of the image content. That allows compensating the motion between different frames and registering across all images of the sequence with a full search block matching algorithm. After the denoising is performed by the averaging and BG subtraction steps, the perspective deformation is corrected to obtain a perfectly square panel. The complete set of steps taken by the post processing of drone-based EL images can be seen in the green box of Fig. 3 .

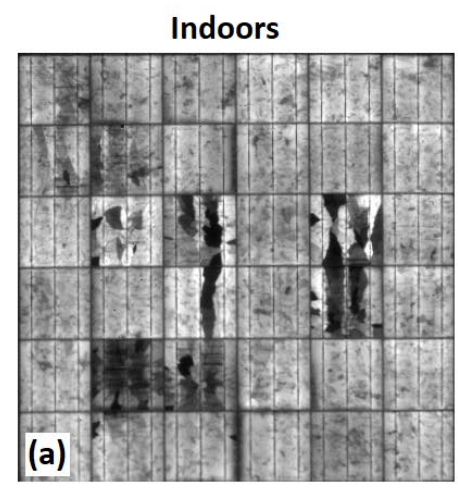

$\mathrm{SNR}_{\mathbf{5 0}}=\mathbf{2 0 . 3}$

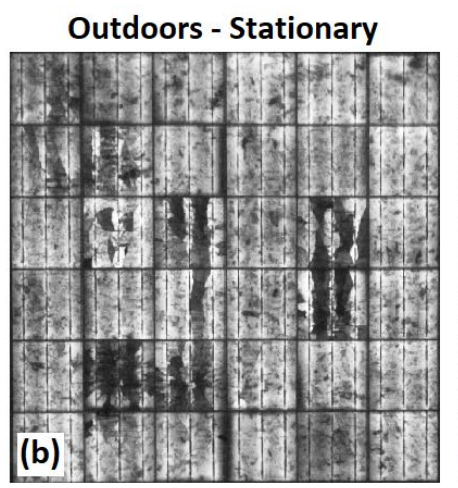

AC+DC modulation $\mathrm{G}_{\mathrm{POA}}=891.0 \mathrm{~W} \mathrm{~m}^{-2}$ $S N R_{A V G}=15.0$
The amount of images needed to improve the SNR until the recommended quality depends of many empirical factors, such as sun irradiance and EL/BG difference, but the main and the most decisive factor for image improvement is spatial match [7]. When spatial match is not a problem (in the case of stationary daylight EL), 16 image pairs are enough for visually acceptable EL image for cracks diagnosis [6]. In this work, we are dealing with two extreme scenarios, which are high sun irradiances and motion acquisition. Sequences acquired under high irradiances tend to provide smaller EL/BG deltas, however a considerable number of images (around 50 image pairs) well spatially matched overcomes this drawback and provides a good quality image to detect cell cracks. Detailed study correlating the concurrent factors for in daylight EL image quality are still under development.

\section{RESULTS AND DISCUSSION}

Fig. 7 shows the EL images from the cracked PV panel acquired in four different conditions: indoors under low light (Fig. 7a), outdoors under bright daylight with stationary sequential image acquisition and $\mathrm{AC}+\mathrm{DC}$ electrical modulation (Fig. 7b); outdoors under bright daylight with sequential image acquisition in motion from the drone system and DC electrical modulation (Fig. 7c); and outdoors under bright daylight with sequential images acquired in motion from the drone system and $\mathrm{AC}+\mathrm{DC}$ electrical modulation (Fig. 7d). All outdoor sequences had 100 image pairs acquired and the resulting images required the post processing described in Section II.C, where the motion compensation took part only when the sequences were obtained in motion from the drone.

The drone acquisition of the DC modulated image occurred in a clear sky morning, with GHI varying from 839.9 to 845.7 $\mathrm{W} \mathrm{m} \mathrm{m}^{-2}$ during the course of the data acquisition. The module was positioned facing the sun and had approximately $970.6 \mathrm{~W}$ $\mathrm{m}^{-2}$ in the POA. The drone acquisition of the $\mathrm{AC}+\mathrm{DC}$ modulated image occurred in a partially cloudy afternoon with GHI varying from 226.4 to $768.6 \mathrm{~W} \mathrm{~m}^{-2}$ and with PV panel GPOA measured $856.0 \mathrm{~W} \mathrm{~m}^{-2}$ just before image acquisition. The exposure times in these irradiance situations were $0.37 \mathrm{~ms}$ for Fig. 7b, $0.30 \mathrm{~ms}$ for Fig.7c and $0.26 \mathrm{~ms}$ for Fig $7 \mathrm{~d}$.

Stationary indoor and outdoor EL images (Figs. 7a and 7b
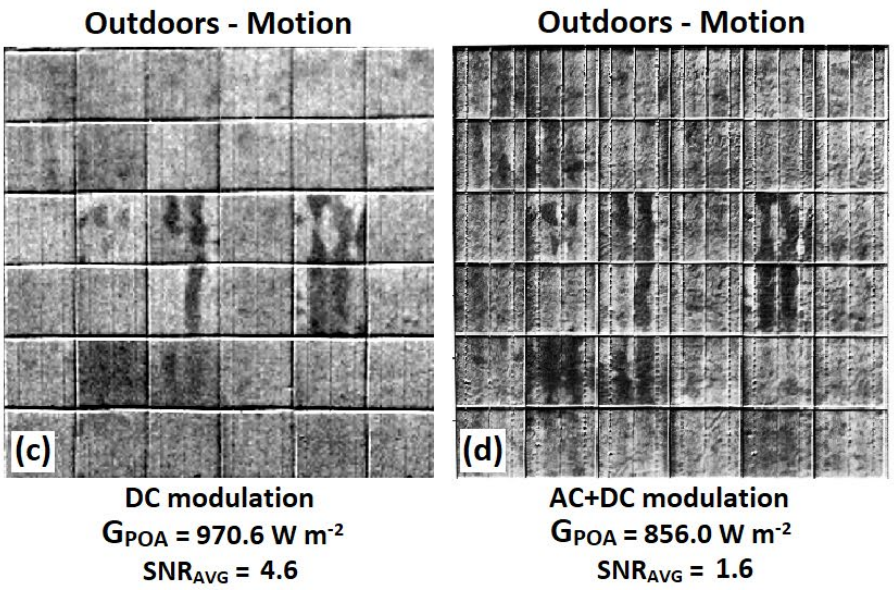

Fig. 7. Mechanically stressed PV panel EL images acquired in different conditions (see text for further details). 
respectively) presented comparable visual quality. The calculated $\mathrm{SNR}_{50}$ and $\mathrm{SNR}_{\mathrm{AVG}}$ were 20.3 for indoor and 15.0 for outdoor stationary respectively. For indoor, this value is relatively low, as it is expected to be higher than 45 for such conditions [4], however, the laboratory conditions was not similar to a dark room environment and the images were acquired with the drone system stationary, where slight motion and shaking may apply and reduce the quality. Here, the indoor image is shown for visual comparison with outdoor images, as the SNR calculations can present visual inconsistencies. The stationary outdoor image is presented for the same reason, as it was acquired in similar GPOA as the images acquired by the drone system.

The EL images obtained in motion from the drone had $\mathrm{SNR}_{\mathrm{AVG}}$ of 4.6 for DC modulation signal and 1.6 for $\mathrm{AC}+\mathrm{DC}$ modulation signal. These values are lower than the minimum requested quality for outdoor EL imaging $\left(\mathrm{SNR}_{50}=5\right)$ [4], while for the DC modulated one is very close. Visually, only $\mathrm{B}$ and $\mathrm{C}$ mode cracks were well identified for both images and the quality of the DC modulated image seems lower than the $\mathrm{AC}+\mathrm{DC}$ modulated, even though the quantified quality tells the contrary. Spatial misalignment can be observed in the cell edges in the form of extra bright or dark edges. This occurred due to the segmentation registration occurring only at pixel level, as sub-pixel would imply interpolation and therefore create other types of uncertainties/mismatches. In Fig. 7c, instabilities in the DC modulation signal summed with the drone motion effected considerably the delta between EL and BG images, causing several images from the sequence to be discarded and there were fewer images available to improve quality. The visually low quality level presented by Fig. 7c compromises the identification of A cracks. Also, disconnected regions smaller than crystallographic defects cannot be visually identified as cracks. However, disconnected regions that are considerably larger than crystallographic defects are still well distinguable. These faults are the ones that affect the most the power of this mechanically stressed module and can be segmented for power loss estimation.

In Fig. $7 \mathrm{~d}$, the $\mathrm{AC}+\mathrm{DC}$ modulation signal presented more stability and after motion compensation, the delta between EL and BG images was well preserved and most of the images in the sequence could be used for quality enhancement. The lower quality here, compared with the outdoor stationary, is mainly due to mismatch in positioning the image pairs acquired in motion. We observed that positioning mismatch in sub-pixel level is enough to prevent optimal quality improvement in terms of the $\mathrm{SNR}_{\mathrm{AVG}}$ quantification. Moreover, the magnitude of the delta between EL and BG images was considerably smaller in Fig. 7d than in Fig. 7c, what could be the reason for the low calculated SNR $\mathrm{AVG}$. As well as for Fig. 7c, the A cracks were barely identified in Fig. $7 \mathrm{~d}$, but crystallographic defects and disconnected cracks with similar dimensions are better differentiable than in Fig. 7c.
Improving quality of sequences acquired in motion proved to be a challenge, even with reproducible module segmentation and EL/BG image selection. The main reason we found so far for not reaching visual quality similar to the outdoor stationary was the large area where sub-pixel level matching of images is crucial. Before these results and observations found during the process of reaching them, stable modulation signals under sunlight and improved algorithms to active a higher level of match between the EL/BG image pairs are highly recommended to obtain daylight EL images from drones successfully. Additionally, the method suggested by the EL technical specification [4] to quantify the quality of the EL images presented inconsistencies between the calculation and visual identification of cell cracks.

\section{CONCLUSION}

The drone-based daylight EL system presented in this paper is able to accomplish EL imaging of PV modules during high solar irradiance. The final resulting EL images presented lower quality compared with indoors and stationary daylight EL and enough quality to identify disconnected cell regions larger than crystallographic defects of multicrystalline Si cells. To the best of our knowledge, this was the first time reporting on EL images, which have been acquired from a drone with global horizontal solar irradiance higher than $100 \mathrm{~W} \mathrm{~m}^{-2}$.

We also quantified the EL images quality following the recently published technical specification [4]. For the drone acquired images, the $\mathrm{SNR}_{\mathrm{AVG}}$ presented a clear discrepancy between the number obtained and the visual quality of the EL images, with crack details possible to be identified in the image with an $\mathrm{SNR}_{\mathrm{AVG}}$ of 1.6 were not possible to be distinguished in the image with $\mathrm{SNR}_{\mathrm{AVG}}$ of 4.6. This indicates that a review of the method, especially for daylight EL, should take place in the future.

$\mathrm{AC}+\mathrm{DC}$ electrical modulation offered a more reproducible mean pixel value delta between EL and BG images than DC electrical modulation and DC modulation presented higher delta magnitude than $\mathrm{AC}+\mathrm{DC}$ modulation. The positioning mismatch was still the main issue for low quality in the dronebased EL.

We presented a look into the technical methods implemented so far, with the acquisition of individual module EL images during the day from a drone system. The algorithms demand further programing steps for a drone-based EL acquisition system to work efficiently in a utility scale environment.

\section{ACKNOWLEDGMENTS}

The authors acknowledge the financial support from Innovation Fund Denmark for the project 6154-00012B DronEL - Fast and accurate inspection of large photovoltaic plants using aerial drone imaging. 


\section{REFERENCES}

[1] IEA, “Renewables 2018," 2018. [Online]. Available: https://www.iea.org/media/presentations/Renewables2018Launch-Presentation.pdf. [Accessed: 18-Aug-2019].

[2] M. Köntges et al., "Review on Infrared and Electroluminescence Imaging for PV Field Applications - IEAPVPS, Report," 2017.

[3] S. Koch, T. Weber, T. Sobottka, A. Fladung, P. Clemens, and J. Berghold, "Outdoor Electroluminescence Imaging of Crystalline Photovoltaic Modules: Comparative Study between Manual Ground-Level Inspections and Drone-Based Aerial Surveys," in 32nd European Photovoltaic Solar Energy Conference and Exhibition, 2016, pp. 1736-1740.

[4] IEC, "IEC TS 60904-13 Photovoltaic Devices - Part 13: Electroluminescence of Photovoltaic Modules," http://www.iec.ch/.

[5] L. Stoicescu, M. Reuter, and J. . Werner, "Daysy: Luminescence Imaging of PV Modules in Daylight," 29th Eur. Photovoltaics Sol. Energy Conf. Exhib. Amsterdam, Netherlands, pp. 2553-2554, 2014.

[6] G. A. dos Reis Benatto et al., "Development of outdoor luminescence imaging for drone-based PV array inspection," 44th IEEE Photovolt. Spec. Conf., 2017.

[7] C. Mantel et al., "SNR Study of Outdoor Electroluminescence Images under High Sun Irradiation," in 2018 IEEE 7th World Conference on Photovoltaic Energy Conversion (WCPEC) (A Joint Conference of 45th IEEE PVSC, 28th PVSEC \& 34th EU PVSEC), 2018, pp. 3285-3289.

[8] J. Adams et al., "Non-Stationary Outdoor EL-Measurements with a Fast and Highly Sensitive InGaAs Camera," 32nd Eur. Photovolt. Sol. Energy Conf. Exhib., 2016.
[9] G. A. dos Reis Benatto et al., "Image Processing for daylight Electroluminescence PV Imaging acquired in movement," 35 th EU PVSEC 2018, pp. 2005-2009, 2018.

[10] Datasheet, "Apalis TK1." [Online]. Available: https://docs.toradex.com/103129-apalis-tk1-datasheet.pdf. [Accessed: 18-Aug-2019].

[11] M. Köntges et al., Review of Failures of Photovoltaic Modules. 2014.

[12] Datasheet, "SM 15K - Series 15kW DC POWER SUPPLIES." [Online]. Available: https://www.deltaelektronika.nl/upload/DATA SHEET_SM15K_V201905.pdf. [Accessed: 18-Aug-2019].

[13] T. Kropp, M. Berner, L. Stoicescu, and J. H. Werner, "SelfSourced Daylight Electroluminescence from Photovoltaic Modules," IEEE J. Photovoltaics, vol. 7, no. 5, pp. 1184-1189, 2017.

[14] U. Rau, "Reciprocity relation between photovoltaic quantum efficiency and electroluminescent emission of solar cells," Phys. Rev. B - Condens. Matter Mater. Phys., vol. 76, no. 8, pp. $1-8,2007$.

[15] T. J. Silverman, M. G. Deceglie, K. Vansant, S. Johnston, and I. Repins, "Illuminated outdoor luminescence imaging of photovoltaic modules," in IEEE Photovoltaic Specialists Conference, 2017.

[16] R. Bhoopathy, O. Kunz, M. Juhl, T. Trupke, and Z. Hameiri, "Outdoor photoluminescence imaging of photovoltaic modules with sunlight excitation," Prog. Photovoltaics Res. Appl., no. July, pp. 14-16, 2017.

[17] C. Mantel et al., "Correcting for Perspective Distortion in Electroluminescence Images of Photovoltaic Panels," IEEE 7th World Conf. Photovolt. Energy Convers., 2018. 